\title{
Acceleration in Astrophysical Environments with CR Propa
}

\author{
Aritra Ghosh $^{a}$, Stijn Buitink ${ }^{b}$, Olaf Scholten ${ }^{a, c}$ and Tobias Winchen ${ }^{b *}$ \\ ${ }^{a}$ KVI-CART, University Groningen, P.O. Box 72, 9700 AB Groningen, The Netherlands \\ ${ }^{b}$ Astrophysical Institute, Vrije Universiteit Brussel, Pleinlaan 2, 1050 Brussels, Belgium \\ ${ }^{c}$ Interuniversity Institute for High-Energy, Vrije Universiteit Brussel, Pleinlaan 2, 1050 Brussels, \\ Belgium \\ E-mail: aritraghshogegmail.com, stijn.buitink@vub.be, \\ scholten@kvi.nl, tobias.winchen@rwth-aachen.de
}

\begin{abstract}
CR Propa is a public astrophysical simulation framework for studying propagation and interaction physics of primary and secondary cosmic ray particles. We have incorporated particle acceleration within CR Propa and studied the effects of geometry on the resulting spectral index in diffusive shock acceleration. In this contribution, we present our results and compare them to analytical calculations. In future, this development will allow incorporation of various loss mechanisms, production of secondaries within the simulation model due to pre-existing capabilities of CR Propa.
\end{abstract}

35th International Cosmic Ray Conference - ICRC2017

10-20 July, 2017

Bexco, Busan, Korea

*Presenting Author 


\section{Introduction}

Diffusive Shock Acceleration (DSA) was first proposed in the late 1970s independently by multiple groups [1,2] in the context of acceleration of galactic cosmic rays (CRs). DSA primarily relies on the repeated scatterings of charged particles by magnetic irregularities (for eg. Alfvén waves) frozen inside a magnetic plasma near a shock front. The combination of the energy gain in each shock crossing and the probability of the particle to return to the shock front after each such acceleration cycle results in a power law spectrum with a spectral index of $s=2.0$. The observed spectrum of these particles is $N(E)_{o b s} / d E \propto E^{-2.7}$ and the difference can be explained by a source spectrum $N(E)_{s} / d E \propto E^{-s}$ with $\mathrm{s}$ in the range $2.1 \lesssim s \lesssim 2.4$ combined with energy dependent propagation effects [3]. Besides, for synchrotron emission from non-thermal radio sources such as supernova remnants (SNRs) and lobes of radio galaxies, the spectrum of the $\mathrm{GeV}$ electrons responsible are similar to the one described above with the power law slope in the range $s \sim$ $2.1-2.7$ [3]. Recent developments in astroparticle physics have also resulted in modified versions of DSA being used to explain scenarios beyond its "traditional" domain. In recent years, there have been claims that some of the extraterrestrial Ice Cube neutrinos [4, 5] could have their roots in star-burst regions with multiple powerful winds of young massive stars and SNRs [6].

Numerical studies of DSA were started as early as the 1980s [7] and continue today employing particle-in-cell (PIC) approaches [8]. While the focus of the community has been on macroscopic studies, there have been few attempts to follow the process microscopically due to the long computational times involved. However, in particular studies of the production of secondary messengers during acceleration, microscopic simulations are a necessity. CR Propa 3 [9] is a publicly available simulation tool that combines knowledge about different aspects of CR propagation and interaction - such as large-scale structures, magnetic fields, nuclear decay, particle interaction with background fields etc. and thus can be used to invalidate multiple theoretical scenarios and make progress towards an astrophysical model that can account for all measurements simultaneously.

Incorporation of DSA into CR Propa will allow us to combine the cosmic ray interaction features with acceleration studies. In this work, we present the first incorporation of DSA into CR Propa and demonstrate its robustness by reproducing the results of classic "textbook" DSA. We will also tweak these classic scenarios to study some more realistic situations.

\section{Simulation of DSA}

CR Propa has a modular structure and deals with the various aspects of CR physics via separate independent modules - thus, new modules can be added without having to change the others. For this work, the inbuilt source and propagation modules of CR Propa were used and for the acceleration mechanism and observation/detection, custom modules were added. To simulate DSA, we set up the simulation within a simple box layout as shown in Fig. 1.

Since the simulation is performed in the rest frame of the shock front, the shock front is stationary and is depicted by the grey plane with the shock velocity $U=0$ in Fig. 1 . There are two wire-frame boxes on both sides of the shock front which represent the upstream and downstream regions of the shock. The two XY planes and the two XZ planes are chosen to have periodic boundary conditions and the two pink infinite YZ planes are "plane observers". Considering arguments 


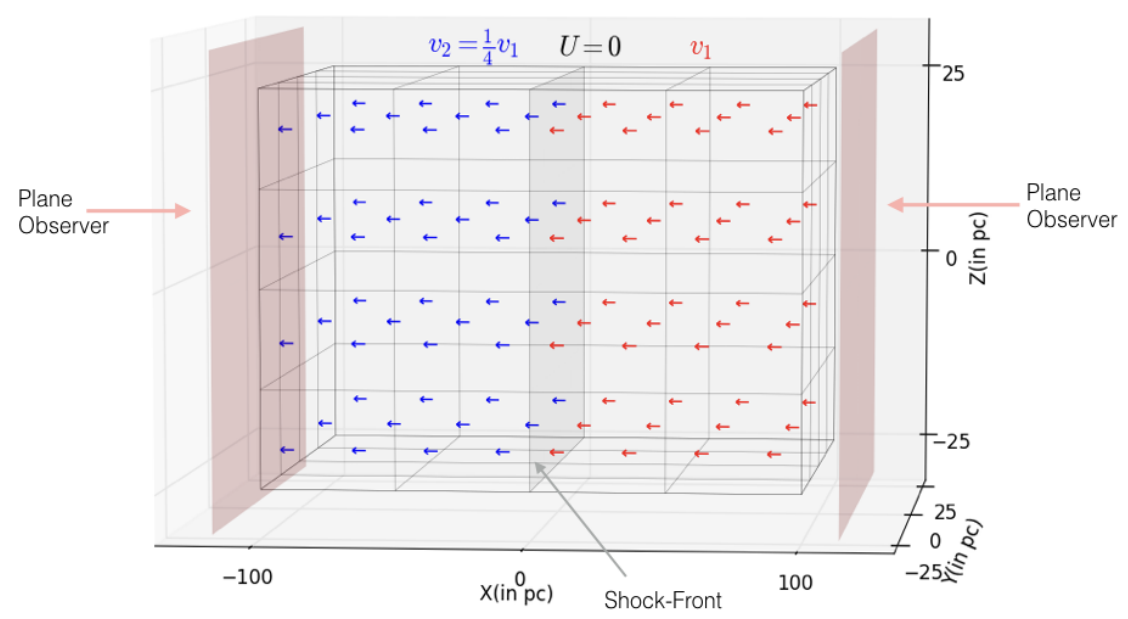

Figure 1: The simulation setup. The pink and the grey planes are the observer planes and the shock front respectively. The arrows depict the movement of the scattering centres on both sides of the shock front.

regarding strong shocks [10], the velocity of the downstream plasma $v_{2}$ is chosen to be $1 / 4$ th of the upstream gas velocity $v_{1}$. The red and the blue arrows depict the motion of the "scattering centres". We assume a plasma of infinite conductivity and hence, via the frozen flux theorem of ideal MHD, the scattering centres move about in the same way as the plasma. Note that while this assumption may not represent the most realistic scenario, it is necessary for reproducing "textbook" DSA.

The particles are injected into the acceleration region from an isotropic source emitting protons with an energy of $10 \mathrm{EeV}$. After that, the simulation progresses with our acceleration module according to the flow chart shown in Fig. 2. The simulation keeps repeating the same cycle until the particle reaches either of the observer planes.

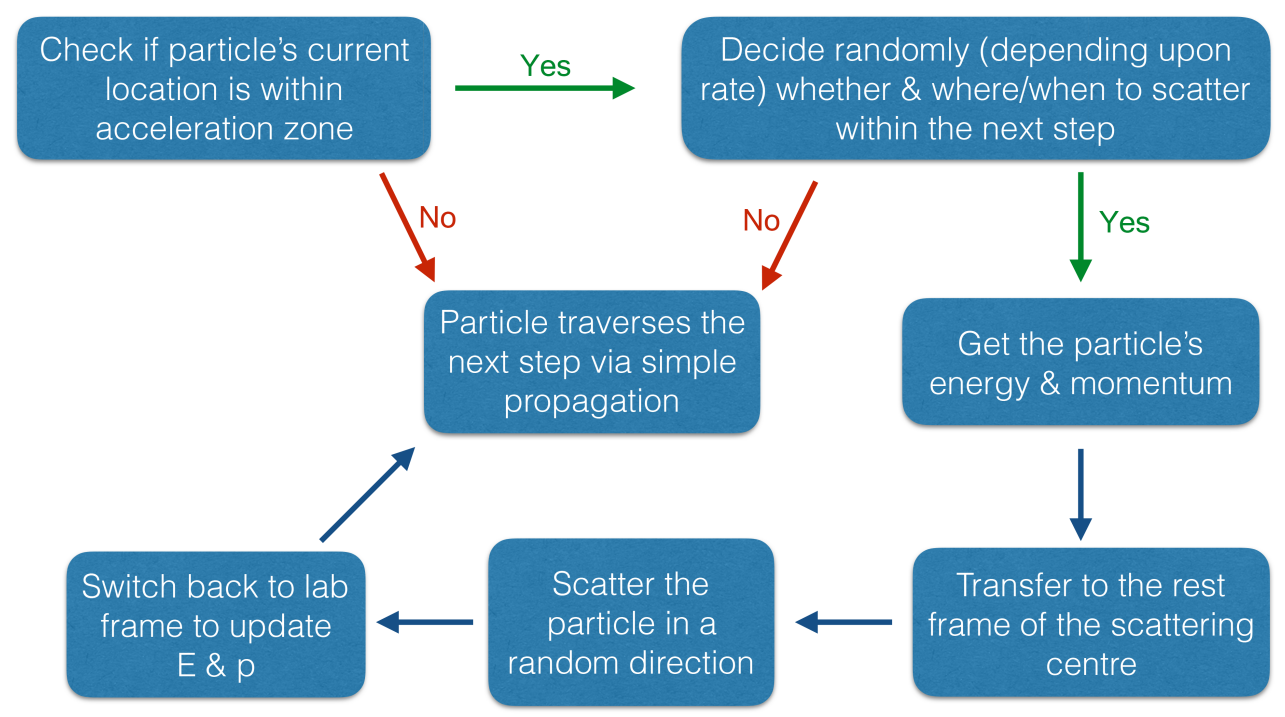

Figure 2: The scheme of operation of the Acceleration Module. 
Therefore in the simulation, the particle is moving around in small steps and changing its direction continuously due to scatterings - it is effectively diffusing around in the simulation volume. During these scatterings, the particles are deflected in a random direction in the rest-frame of the scattering centre and this isotropizes their velocity distribution. By virtue of their motion, the particles cross the shock and whenever they do, they gain energy by Lorentz Transformations [10]. The process described above successfully mimics all aspects of DSA.

The location of the source was moved around in the simulation volume and was found to have no substantial effect on the spectrum obtained. Therefore, to ensure that most particles cross the shock at least once, it is placed in the upstream region and to reduce simulation run time, it is placed close to the shock front at $(30,0,0) \mathrm{pc}$. The mean free path (MFP) between two scatterings is chosen to be $4 \mathrm{pc}$ and the upstream scatter centre velocity/shock velocity as $0.1 \mathrm{c}$. It is almost always assumed in analytical calculations that no particles exit the acceleration region upstream as the particles have the tendency to get advected by the flow of the upstream plasma towards the shock. To make our upstream box long enough while still ensuring realistic run times, we calibrated the setup and obtained an optimum upstream box-size of $L_{r b o x}=250 \mathrm{pc}$.

\section{Effect of Simulation Parameters}

Downstream Box Size First, we investigated the effect of the downstream box size $L_{\text {lbox }}$ on the spectral index. The simulation was performed for various sizes of the downstream box and the results are shown in Fig. 3(a). For $L_{l b o x}=40 \mathrm{pc}$, the index is -2.89 and then it rises rapidly finally appearing to "stabilise" exponentially towards the canonical -2.0 for $L_{l b o x} \geq 250 \mathrm{pc}$.



(a)

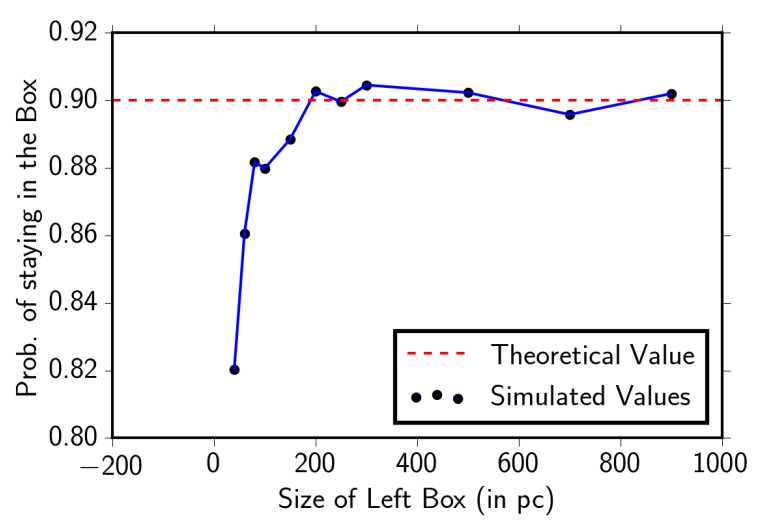

(b)

Figure 3: (a) Spectral Index and (b) Return Probability for various sizes of the downstream box / left box.

The existence of a "critical length" for the downstream box to obtain the canonical spectral index can be connected to the return probability of the particle. If we place the left observer too close to the shock front, then a particle which might have otherwise returned to the shock is now removed from the simulation. When the left box is made long enough, at large distances from the shock, the return probability of the particle approaches zero and removing it does not have a substantial effect. This is shown by Fig. 3(b). The return probability increases with an increase in 
$L_{l b o x}$ before stabilising near the "critical length". For all simulations hereafter, it should be assumed that a stable value of $L_{l b o x}$ (i.e. $L_{l b o x} \geq 400 \mathrm{pc}$ ) has been chosen unless mentioned otherwise.

Average Energy Gain and Return Probability The index of the power law in DSA is given by $-1+(\ln P / \ln \beta)[10]$ where $\beta$ is the average energy increase of the particle after one shock crossing and $\mathrm{P}$ is the probability that the particle remains within the acceleration region after one crossing. $\beta$ and $\mathrm{P}$ vary with the velocity of the shock as $\beta=1+U / c$ and $P=1-U / c$. To verify that $\beta$ and $\mathrm{P}$ vary as expected, the simulation is repeated for various values of the shock velocity and the results are shown in Fig. 4

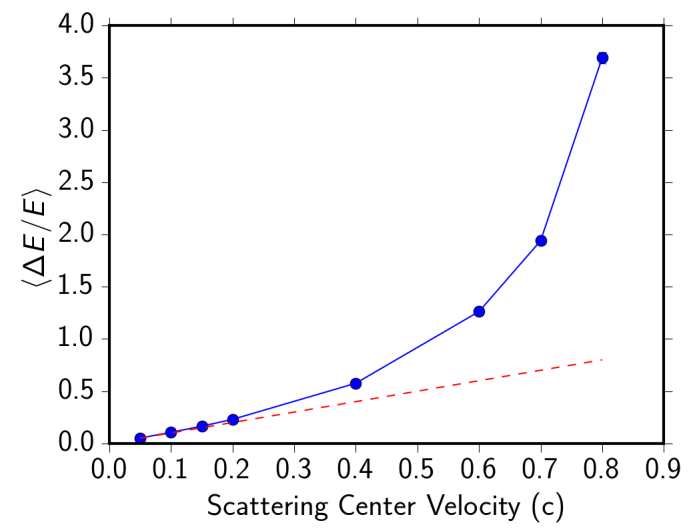

(a)



(b)

Figure 4: (a) Mean value of relative energy gain $(\Delta E / E)$ and (b) probability of the particle to not escape from the box for various values of the upstream scattering centre velocity i.e. the shock velocity. The red-dotted line refers to the theoretically expected values for non-relativistic shocks.

The simulated values are close to the theoretical predictions for smaller shock velocities, but show significant divergence for higher values. Increasing the shock velocity moves us into the domain of relativistic and ultra-relativistic shocks and the usual analytical description does not hold anymore. In such cases, the anisotropy in the angular distribution of the accelerated particles is so large upstream that the spatial diffusion approximation breaks down [3] and hence, our treatment of DSA does not apply anymore. Thus, our simulation is consistent with these expectations and shows that the relativistic effects clearly become visible for values of shock velocity greater than $0.4 \mathrm{c}$.

Energy Dependant Diffusion and Shock Geometry It has been established above that the results of our simulation are consistent with the picture of standard "textbook" DSA. However, the MFP and hence, the scattering rate, scales with the energy of the particle, which is ignored in the canonical calculation. It has been demonstrated in Ref. [11, 12] that for CRs travelling through a background medium that moves with a non-relativistic bulk speed parallel to an ordered magnetic field $B_{0}$ with superposed Alfvén waves propagating parallel and/or anti-parallel to $B_{0}$, the MFP $(\lambda)$ for a particle with energy $\mathrm{E}$ and charge $\mathrm{Z}$ varies as $\lambda=\lambda_{0}\left(\frac{E}{1 \mathrm{EeV}} \frac{1}{\mathrm{Z}}\right)^{2-q}$ where $\mathrm{q}$ is the turbulence index of the magnetic field. 
So far, we have assumed that scattering centres are present uniformly throughout the acceleration region - however, this may very well not be the case in a real-life scenario. Let us assume a Gaussian distribution of scattering centres centred around the shock front. In a sense, under this scheme, the shock now has a structure and a definite "width". Let the shock-width be defined as the standard deviation $\sigma$ of the Gaussian distribution of scattering centres.

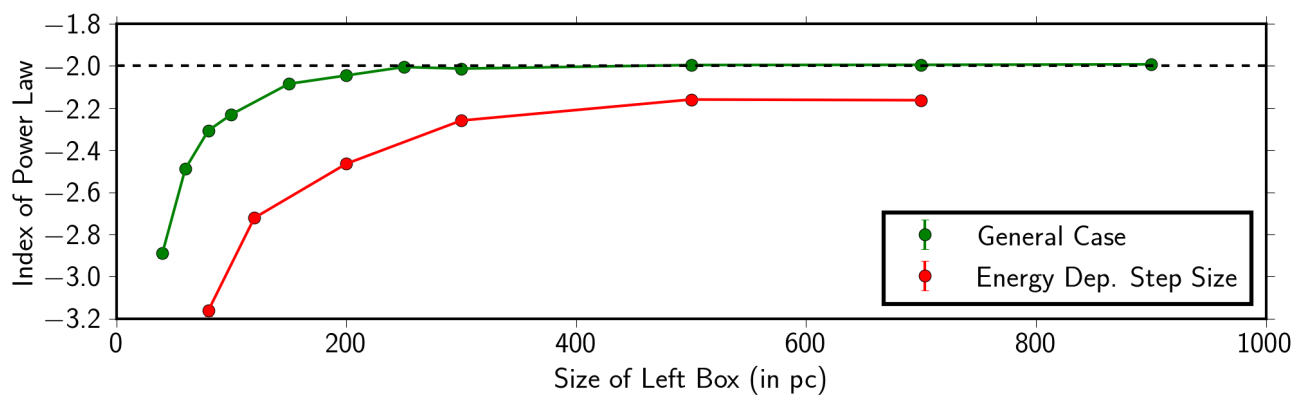

(a)



(b)



(c)

Figure 5: The indexes of the power law spectrum for (a) an energy dependent scattering rate, (b) for different shock widths and (c) for different shock widths with an energy dependent scattering rate. The corresponding percentages in the legend box of (b) and (c) refer to the percentage of total particles that are detected by the upstream observer.

Figs. 5(a) and 5(b) show the power law indexes obtained for an energy dependant step length and for Gaussian distributions of scattering centres with various standard deviations respectively. The nature of the variation in both cases is the same as that of the textbook case. However, the curves now level off to lower values instead of -2 . For an energy dependent MFP, as the particle gains more energy it travels in larger steps and with a Gaussian distribution of scattering centres, 
particles encounter fewer scattering centres as they move away from the shock front - both of these effects alter the escape probability of the particle and hence the results differ from textbook DSA. Simulations were also performed with both these effects combined and the results are shown in Fig. 5(c). With both the features combined, the effect on the simulation was more pronounced, producing even more softer spectra than one might expect by a linear addition of the two effects. Fig. 6(a) shows the indexes of the power law for various shock-widths for $L_{l b o x}=400 \mathrm{pc}$. Therefore, these are the index values which are asymptotically approached by each of the corresponding spectra. This "levelling off" value increases with shock-width for both cases as shown in Fig. 6(a) with the energy-independent case seeming to reach the standard -2.0 for an infinite shock-width.

The varying distribution of scattering centres also has an effect on the situation in the upstream box. As the shock becomes narrower, a large number of the scattering centres become concentrated in a small region near the shock and once a particle has been able to make its way through this dense region, there is almost nothing stopping it from exiting the simulation volume using the upstream exit. For this purpose, to ensure lower rates of particle leakage, $L_{r b o x}$ is increased to $400 \mathrm{pc}$ for all these simulations. Even with this, in many cases the particle leakage rate was greater than $1 \%$.

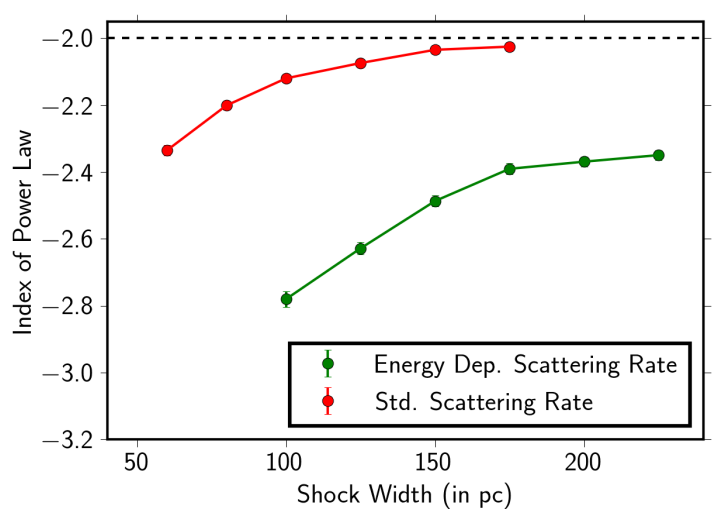

(a)

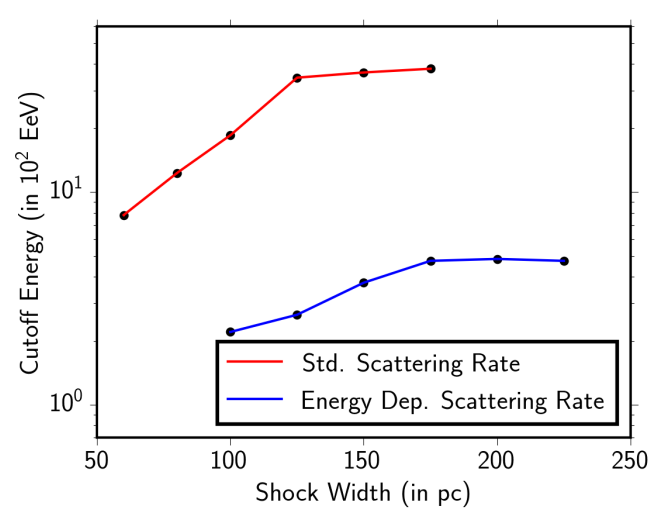

(b)

Figure 6: (a) The indexes of the power law and (b) the cut-off energy for various shock widths.

In the textbook simulation, particles are always accelerated almost up to $10^{4} \mathrm{EeV}$ (with an injection energy of $10 \mathrm{EeV}$ ) for stable downstream box-sizes. But, the spectrum for the cases with a Gaussian distribution of scattering centres have a cut-off much earlier in the energy range and the location of the cut-off depends on the shock-width and whether the scattering rate is energy dependent or not as shown in Fig. 6(b). The maximum energy to which a particle can be accelerated depends on the time the particle spends inside the acceleration zone. Since a Gaussian distribution of scattering centres alters the escape probability, it also alters the maximum energy to which particles can be accelerated. Therefore, Fig. 6(b) alludes to the fact that a wider shock leads to a lower escape probability.

\section{Conclusion}

We presented the first study of DSA with CR Propa. We reproduced the standard DSA spec- 
trum with a spectral index of -2.0 and also investigated limiting cases yielding softer spectra. The relation of the spectrum with various geometrical parameters such as $L_{l b o x}$ and $L_{r b o x}$ was analyzed, giving us insight into how the geometry of the acceleration region affects the particle spectrum. We studied the energy gain factor and return probability which showed agreement with theoretical predictions. We also demonstrated that the simple treatment of DSA is no longer valid for (ultra) relativistic shocks and that results start showing significant deviation for shock velocities greater than $0.4 \mathrm{c}$. At the end, we looked at two ways the simulation can be modified to include more detailed effects beyond "textbook" DSA.

By incorporating DSA within CR Propa, we have set up the base for new microscopic simulations of DSA. CR Propa has in-built modules to simulate energy losses by interaction with background photons, expansion of the universe and create secondary nuclei, photons, neutrinos from various interactions and decays. By combining the acceleration module described herein with these pre-existing modules, we open up the possibility to study these scenarios.

\section{References}

[1] A. R. Bell. The acceleration of cosmic rays in shock fronts. I. MNRAS, 182 147, 1978.

[2] R. D. Blandford and J. P. Ostriker. Particle acceleration by astrophysical shocks. ApJL, 221 L29, 1978.

[3] A. Achterberg et al. Particle acceleration by ultrarelativistic shocks: theory and simulations. MNRAS, 328 (2) 393, 2001.

[4] M. Aartsen et al. Observation of High-Energy Astrophysical Neutrinos in Three Years of IceCube Data. PRL, 113 101101, 2014.

[5] M. Aartsen et al. Search for correlations between the arrival directions of IceCube neutrino events and ultrahigh-energy cosmic rays detected by the Pierre Auger Observatory and the Telescope Array. JCAP, 2016 (01) 037, 2016.

[6] A. Bykov et al. Ultrahard spectra of PeV neutrinos from supernovae in compact star clusters. MNRAS, 453 (1) 113, 2015.

[7] C. M. Ko and J. R. Jokipii. A numerical study of diffusive shock acceleration of cosmic rays in supernova shocks. Proceedings of the ICRC, 1985.

[8] H. Kang and T. Jones. Numerical studies of diffusive shock acceleration at spherical shocks. AP, 25 (4) $246,2006$.

[9] R. A. Batista et al. CRPropa 3-a public astrophysical simulation framework for propagating extraterrestrial ultra-high energy particles. JCAP, 2016 (05) 038, 2016.

[10] M. Longair. High Energy Astrophysics. Cambridge University Press, 2011.

[11] R. Schlickeiser. Cosmic-ray transport and acceleration. II. Cosmic rays in moving cold media with application to diffusive shock wave acceleration. ApJ, 336 264, 1989.

[12] J. Scalo and B. G. Elmegreen. Interstellar turbulence II: Implications and effects. ARAA, 42 275, 2004. 